Revista Brasileira de Agricultura Irrigada v.8, nº. 2, p. 154 - 160, 2014

ISSN 1982-7679 (On-line)

Fortaleza, CE, INOVAGRI - http://www.inovagri.org.br

DOI: $10.7127 /$ rbai.v8n200241

Protocolo 241/14 - 14/09/2013 Aprovado em 31/03/2014

\title{
COMPORTAMENTO VEGETATIVO DO FEIJÃO CAUPI IRRIGADO E ADUBADO SOB DIFERENTES DOSES DE BIOFERTILIZANTE ORGÂNICO
}

\author{
Joscélia da Silva Cruz; Edgleudo Coelho de Sousa²; José Aguiar Beltrão Júnior³; Juliana \\ Martins Uchôa de Almeida ${ }^{4}$; Neyreane de Sousa Luna ${ }^{5}$
}

\begin{abstract}
RESUMO
O feijão caupi constitui-se em um dos principais componentes da dieta alimentar nas regiões Nordeste e Norte do Brasil, especialmente na zona rural. No estado do Ceará esta cultura detém a maior parte das áreas de cultivo, porém a deficiência nutricional é considerada um dos principais fatores atribuídos ao baixo rendimento da cultura nos solos do litoral do estado. O objetivo deste trabalho foi avaliar o desenvolvimento do feijão caupi em função de diferentes doses do biofertilizante orgânico, produzido através da biodegradação acelerada dos resíduos da palha e inflorescências do coqueiro, poda de nim e poda de cajueiro comparado à adubação mineral, nos diferentes estádios de desenvolvimento do mesmo. $\mathrm{O}$ delineamento adotado foi de blocos casualizados com cinco tratamentos e quatro repetições sendo quatro tratamentos constituídos de diferentes doses do biofertilizante orgânico $(0 \mathrm{~L}, 2 \mathrm{~L}$, $4 \mathrm{~L}$ e $6 \mathrm{~L} /$ parcela de $3 \mathrm{~m}$ linear) e um com adubação química. As vaiáveis analisadas foram: altura da planta, diâmetro do caule e diâmetro da copa. A adubação química proporcionou maiores valores em relação as doses de biofertilizante orgânico aplicado na cultura se tratando das variáveis analisadas.
\end{abstract}

Palavras-chave: Vigna Unguiculata L, biofertilizante orgânico, desenvolvimento vegetativo

\section{BEHAVIOR VEGETATIVE OF IRRIGATED COWPEA AND FERTILIZED CROP UNDER DIFFERENT DOSES OF ORGANIC BIOFERTILIZER}

\footnotetext{
ABSTRACT

The cowpea is itself one of the main components of the diet in the North and Northeast regions of Brazil, especially in the countryside. In the state of Ceará, this crop is grown in most growing areas; however, its nutritional deficiency is considered one of the main factors attributed to the low crop yield in soils of the coastal state. The objective of this study was to evaluate the development of cowpea due to different doses of organic bio fertilizer, produced

${ }^{1}$ Tecnóloga em Irrigação e Drenagem, Pesquisadora do Instituto INOVAGRI, Fortaleza - CE. E-mail: joscelia@inovagri.org.br

${ }^{2}$ Tecnólogo em Irrigação e Drenagem, Pesquisador do Instituto INOVAGRI, Fortaleza - CE.

${ }^{3}$ Especialista em Irrigação e Drenagem, Pesquisador do Instituto INOVAGRI, Fortaleza - CE.

${ }^{4}$ Tecnóloga em Agronegócio, Pesquisadora do Instituto INOVAGRI, Fortaleza - CE.

${ }^{5}$ Tecnóloga em Irrigação e Drenagem, Pesquisadora do Instituto INOVAGRI, Fortaleza - CE.
} 
by accelerated biodegradation of waste straw and inflorescence of coconut, Nim pruning and Cashew Tree pruning, compared to mineral fertilizer at different stages of development thereof. The design was a complete randomized block with five treatments and four replications, with four treatments consisting of different doses of organic bio fertilizer (0L, $2 \mathrm{~L}, 4 \mathrm{~L}$ and $6 \mathrm{~L} /$ parcel $3 \mathrm{~m}$ linear) and one with chemical fertilizer. The variables analyzed were: plant height, stem diameter and crown diameter. The chemical fertilizer gave higher values compared with doses of organic bio fertilizer applied to the crop in the case of the variables analyzed.

Keywords: Vigna unguiculata L, organic bio fertilizers, plant growth

\section{INTRODUÇÃO}

O feijão caupi é originário da África, de onde disseminou para as regiões tropicais dos continentes americano, asiático e africano (SILVA et al., 2008). Conhecido no Nordeste Brasileiro por feijão-de-corda é uma das principais culturas desta região, pois tem bom desenvolvimento em regiões com solos pobres em nutrientes, além de tolerar estresse hídrico (OLIVEIRA et al., 2013).

No estado do Ceará esta cultura detém a maior parte das áreas de cultivo, sendo uma das principais culturas usadas nos consórcio em áreas irrigadas com culturas perenes, pois além do retorno financeiro auxilia na fixação de nutriente ao solo, como exemplo o consórcio coco x feijão, amplamente praticado no litoral do estado. Porém a deficiência nutricional é considerada um dos principais fatores atribuídos ao baixo rendimento do cultivo do feijão caupi nos solos do litoral do estado. Para reduzir estes problemas, pesquisadores vêm buscando alternativas que proporcionem elevação da produtividade com baixo impacto ao ambiente. Uma delas é emprego do composto orgânico que auxilia na manutenção da fertilidade do solo, pela liberação de nutrientes no processo de decomposição de maneira graduada (BARROS \& LIBERANO FILHO, 2008).

O uso de compostos orgânicos a base de biofertilizantes vêm sendo utilizada no cultivo de espécies agrícola, como por exemplo, Lima et al. (2012), avaliando o crescimento inicial do milho fertirrigado com biofertilizante, concluíram que o biofertilizante bovino estimulou a produção de matéria seca da folha, do colmo e da parte aérea do milho aos sessenta dias após o plantio. A riqueza nutricional e biológica que os compostos orgânicos conferem ao solo e as plantas auxiliam sobre maneira no seu cultivo, permitindo melhorar as qualidades químicas, físicas e biológicas do solo (MELO et al., 2007).

Objetivou-se com essa pesquisa avaliar a altura do feijão caupi em função de diferentes doses do biofertilizante orgânico, produzido através da biodegradação acelerada dos resíduos da palha e inflorescências do coqueiro, poda de nim e poda de cajueiro, comparado à adubação mineral, nos diferentes estádios de desenvolvimento do mesmo. 


\section{MATERIAL E MÉTODOS}

O experimento foi conduzido na fazenda experimental do Instituto de Pesquisa e Inovação na Agricultura Irrigada - INOVAGRI, localizada no município de Trairí no litoral oeste do estado a $125 \mathrm{~km}$ de Fortaleza, em delineamento experimental de blocos casualizados, com quatro repetições e cinco tratamentos sendo um deles uma testemunha com adubação mineral. Os tratamentos foram constituídos de diferentes tipos de adubo (Tabela 1). As parcelas constituíram de 30 plantas, espaçadas de $0,5 \mathrm{~m}$ entre planta e $1,0 \mathrm{~m}$ entre fileiras, sendo 06 na parcela útil para análises dos resultados. A análise do solo indicou a seguinte composição: $\mathrm{pH}=5,6\left(\mathrm{cmol}_{\mathrm{c}} / \mathrm{kg}\right) ; \mathrm{Ca}^{2^{+}}=0,50\left(\mathrm{cmol}_{\mathrm{c}} / \mathrm{kg}\right) ;$ $\mathrm{Mg}^{2^{+}}=0,40\left(\mathrm{cmol}_{\mathrm{c}} / \mathrm{kg}\right) ; \mathrm{Na}^{+}=0,03\left(\mathrm{cmol}_{\mathrm{c}} / \mathrm{kg}\right) ;$ $\mathrm{K}^{+}=0,04\left(\mathrm{cmol}_{\mathcal{C}} / \mathrm{kg}\right) ; \mathrm{H}^{+}+\mathrm{Al}^{3^{+}}=1,32\left(\mathrm{cmol}_{\mathrm{C}} / \mathrm{kg}\right)$; $\mathrm{Al}^{3^{+}}=0,15\left(\mathrm{cmol}_{\mathrm{c}} / \mathrm{kg}\right) ; \mathrm{C}=3,2(\mathrm{~g} / \mathrm{kg}) ; \mathrm{N}=0,27$ $(\mathrm{g} / \mathrm{kg}) ; \mathrm{C} / \mathrm{N}=12$ e Matéria orgânica $=5,38$ $(\mathrm{g} / \mathrm{kg})$. O biofertilizante orgânico obteve as seguintes características: $\mathrm{N}=8,1(\mathrm{~g} / \mathrm{Kg}) ; \mathrm{P}=$ $15,8(\mathrm{~g} / \mathrm{Kg}) ; \mathrm{P} 2 \mathrm{O} 5=36,2(\mathrm{~g} / \mathrm{Kg}) ; \mathrm{K}=6,0$ $(\mathrm{g} / \mathrm{Kg}) ; \mathrm{Ca}=66,8(\mathrm{~g} / \mathrm{Kg}) ; \mathrm{Mg}=14,6(\mathrm{~g} / \mathrm{Kg}) ; \mathrm{Fe}$ $=652,6(\mathrm{mg} / \mathrm{kg}) ; \mathrm{Cu}=6,7(\mathrm{mg} / \mathrm{kg}) ; \mathrm{Zn}=29,3$ $(\mathrm{mg} / \mathrm{kg}) ;$ umidade $=49,1 \% ; \mathrm{CO}=347,4(\mathrm{~g} / \mathrm{kg})$ e $\mathrm{C} / \mathrm{N}=42,9$.

O preparo do solo foi feito através de gradagens e calagem para correção do $\mathrm{pH}$. As doses de adubo foram aplicadas na linha de plantio em forma de suco e as sementes foram cultivadas em covas ao lado do adubo, sentido contrário da declividade do relevo. A adubação mineral foi seguida de acordo com os resultados da análise de solo com a seguinte recomendação: $25 \mathrm{~kg} / \mathrm{ha}$ de uréia na fundação e $25 \mathrm{~kg} / \mathrm{ha}$ na cobertura com 30 dias após a emergência; 100 $\mathrm{kg} / \mathrm{ha}$ de P2O5 (usando fosfato monoamônico ou MAP) e $90 \mathrm{~kg} / \mathrm{ha}$ de Cloreto de Potássio. Durante a condução da cultura foram realizadas inspeções diárias, nas quais foram observadas ataques de algumas pragas, para isso foi realizado o controle a base de defensivos químicos: Deltametrina 2,5E $(06 \mathrm{ml}) \quad \mathrm{e}$
Abamectina $(24 \mathrm{ml})$ para controle de cigarrinhas (Empoasca kraemeri) e mosca-branca (Bemisia argentifolii); Agrinose (200g) para controle de ferrugem (Uromyces appendiculatus) e antracnose (Colletotrichum lindemuthianum).

Foram realizadas também capinas com auxílio de enxadas, procurando-se manter a cultura sempre livre de plantas invasoras. A irrigação era realizada com sistema de microaspersão e turno de rega de um dia.

Foram realizadas leituras com 20, 40 e 60 dias após a germinação, para análises das variáveis: altura da planta, diâmetro do caule e diâmetro da copa.

Tabela 1. Tratamentos que foram empregados no experimento

\begin{tabular}{cc}
\hline Tratamentos & Tipo de Adubo \\
\hline T0 & Testemunha (adubação química) \\
T1 & L/m Linear do adubo \\
T2 & $2 \mathrm{~L} / \mathrm{m}$ linear do Adubo \\
& Biotecnológico \\
T3 & $4 \mathrm{~L} / \mathrm{m}$ linear do Adubo \\
& Biotecnológico \\
T4 & $6 \mathrm{~L} / \mathrm{m}$ linear do Adubo \\
& Biotecnológico \\
\hline
\end{tabular}

\section{RESULTADO E DISCUSSÃO}

De acordo com a tabela 2 pode-se observar que não houve efeito significativo da aplicação de diferentes doses do composto orgânico entre si, em comparação com a adubação mineral com 20 e 40 dias para variável altura da planta. Já para o tratamento (testemunha) com adubação mineral houve elevação da altura da planta com 20 e 40 dias, porém com 60 dias não houve 
efeito positivo para adubação química (Fig. 1). Todos os resultados são inferiores dos encontrados por Simone et al (2009) ao analisar desempenho da cultura sob diferentes doses de biofertilizantes aplicado no solo.

Tabela 2. Resumo da análise de variância para altura de planta aos 20, 40 e 60 dias das plantas submetidas a $0,2,4,6$ e adubação química.

\begin{tabular}{ccccc}
\hline \multirow{2}{*}{$\begin{array}{c}\text { Fonte de } \\
\text { Variação }\end{array}$} & GL & \multicolumn{3}{c}{ Quadrados Médio } \\
\cline { 3 - 5 } Tratamento & 4 & $161,43^{* *}$ & $515,67^{* *}$ & $211,08^{* *}$ \\
Bloco & 3 & $4,58 \mathrm{~ns}$ & $122,18^{* *}$ & $30,47 \mathrm{~ns}$ \\
Resíduo & 12 & 95,50 & 13,31 & 25,68 \\
CV & & 9,39 & 9,11 & 13,03 \\
\hline Tratamentos & & & Médias $(\mathrm{cm})$ \\
\cline { 2 - 4 } 0 & & $31,00 \mathrm{~b}$ & $42,25 \mathrm{~b}$ & $41,50 \mathrm{a}$ \\
2 & $29,25 \mathrm{~b}$ & $35,50 \mathrm{~b}$ & $38,25 \mathrm{a}$ \\
4 & $28,75 \mathrm{~b}$ & $40,75 \mathrm{~b}$ & $40,50 \mathrm{a}$ \\
6 & $21,75 \mathrm{~b}$ & $25,25 \mathrm{~b}$ & $27,25 \mathrm{~b}$ \\
Testemunha & & $39,50^{\mathrm{a}}$ & $56,50 \mathrm{a}$ & 47,00 \\
\hline
\end{tabular}

** e * significativo a 1 e $5 \%$ de probabilidade pelo teste $\mathrm{F}$, respectivamente; ns - não significativo a $5 \%$ de probabilidade pelo teste F. Médias seguidas de mesma letra não diferem entre si ao nível de $5 \%$ de probabilidade pelo teste de Dunnett.

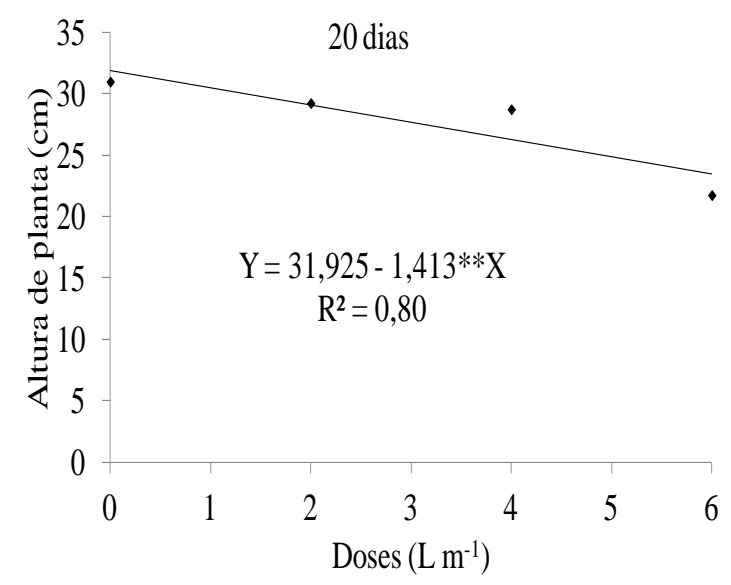

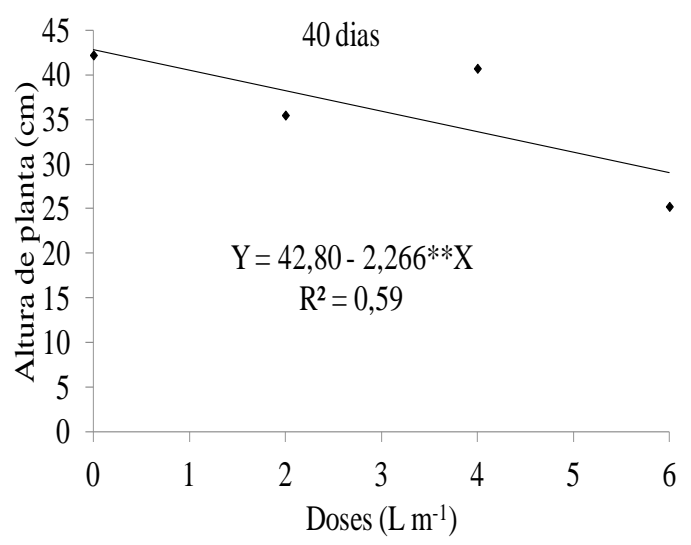

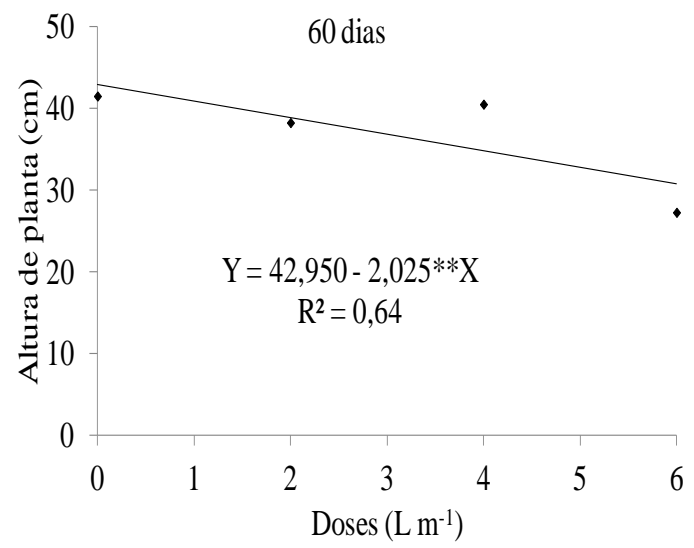

Figura 1. Altura de planta do feijoeiro aos 20, 40 e 60 dias em função a diferentes doses do composto orgânico.

Ao analisar a variável diâmetro do caule (tabela 3) observou-se que não houve diferença significativa entre si nos tratamentos utilizados com o adubo orgânico pra nas três fases avaliadas (20, 40 e 60 dias), no entanto para adubação mineral houve resultado positivo nas três fases quando comparado com adubação orgânica com diferentes doses do composto (Fig.2). Sousa et al. (2013) obtiveram efeito significativo sobre o diâmetro do caule de pinhão-manso ao aplicar biofertilizante bovino até aos 80 dias após o transplantio. 
Tabela 3. Resumo da análise de variância para o diâmetro de caule aos 20, 40 e 60 dias das plantas submetidas a $0,2,4,6$ e adubação química.

\begin{tabular}{ccccc}
\hline Fonte de & \multirow{2}{*}{ GL } & \multicolumn{3}{c}{ Quadrados Médio } \\
\cline { 3 - 5 } Variação & & 20 dias & 40 dias & 60 dias \\
\hline Tratamento & 4 & $7,25^{* *}$ & $21,95^{* *}$ & $23,88^{* *}$ \\
Bloco & 3 & $0,58 \mathrm{~ns}$ & $1,65 \mathrm{~ns}$ & $2,18^{*}$ \\
Resíduo & 12 & 0,25 & 0,65 & 0,48 \\
CV & & 11,76 & 13,11 & 11,03 \\
\hline Tratamentos & \multicolumn{3}{c}{ Médias $(\mathrm{mm})$} \\
\cline { 3 - 5 } 0 & & $4,35 \mathrm{~b}$ & $6,00 \mathrm{~b}$ & $6,25 \mathrm{~b}$ \\
2 & $3,50 \mathrm{~b}$ & $5,00 \mathrm{~b}$ & $5,00 \mathrm{~b}$ \\
4 & & $4,00 \mathrm{~b}$ & $6,00 \mathrm{~b}$ & $6,00 \mathrm{~b}$ \\
6 & $3,00 \mathrm{~b}$ & $3,75 \mathrm{~b}$ & $3,75 \mathrm{~b}$ \\
Testemunha & $6,50^{\mathrm{a}}$ & $10,00 \mathrm{a}$ & $10,25^{\mathrm{a}}$ \\
\hline
\end{tabular}

** e * significativo a 1 e $5 \%$ de probabilidade pelo teste $\mathrm{F}$, respectivamente; ns - não significativo a $5 \%$ de probabilidade pelo teste $\mathrm{F}$. Médias seguidas de mesma letra não diferem entre si ao nível de $5 \%$ de probabilidade pelo teste de Dunnett

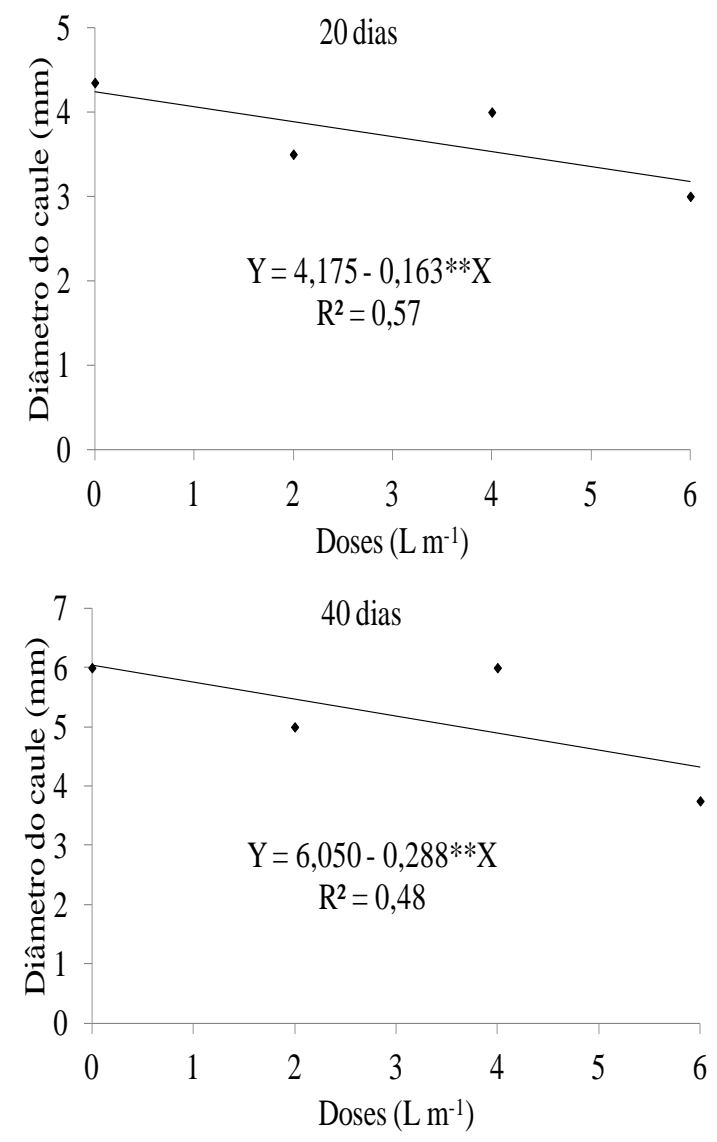

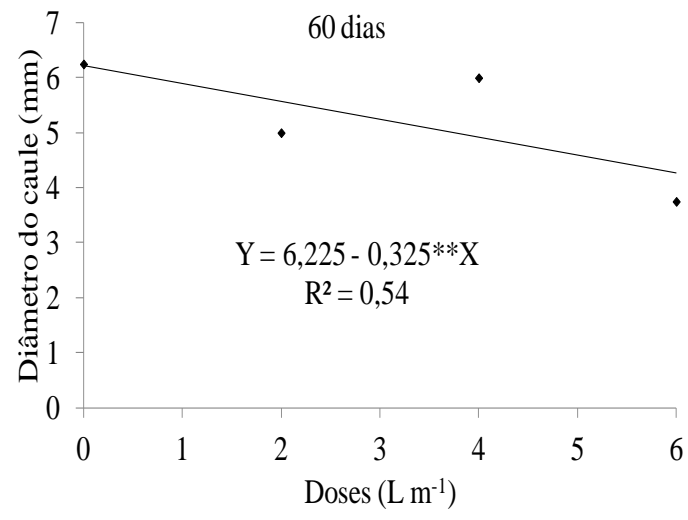

Figura 2. Diâmetro do caule do feijoeiro aos 20, 40 e 60 dias em função a diferentes doses do composto orgânico.

Com relação ao diâmetro da copa observose um efeito significativo nas fases com 20 e 40 dias (tabela 4) para adubação mineral comparada com adubação orgânica. Já na fase com 60 dias não houve diferença significativa para todos os tratamentos, ou seja, a adubação orgânica foi semelhante á adubação química não diferenciando entre si nos resultados (Fig. 3).

Tabela 4. Resumo da análise de variância para o diâmetro da copa aos 20, 40 e 60 dias das plantas submetidas a $0,2,4,6$ e adubação química.

\begin{tabular}{|c|c|c|c|c|}
\hline \multirow{2}{*}{$\begin{array}{l}\text { Fonte de } \\
\text { Variação }\end{array}$} & \multirow{2}{*}{ GL } & \multicolumn{3}{|c|}{ Quadrados Médio } \\
\hline & & 20 dias & 40 dias & 60 dias \\
\hline Tratamento & 4 & $160,93 * *$ & $515,93 * *$ & $55,93 \mathrm{~ns}$ \\
\hline Bloco & 3 & $14,47 \mathrm{~ns}$ & $82,33 \mathrm{~ns}$ & $26,98 \mathrm{~ns}$ \\
\hline Resíduo & 12 & 4,93 & 22,13 & 27,03 \\
\hline $\mathrm{CV}$ & & 6,79 & 11,85 & 16,27 \\
\hline Tratamentos & & \multicolumn{3}{|c|}{ Médias (mm) } \\
\hline 0 & & $32,00 \mathrm{~b}$ & $40,75 b$ & $33,75 a$ \\
\hline 2 & & $30,25 b$ & $34,00 \mathrm{~b}$ & $30,50 \mathrm{a}$ \\
\hline 4 & & $32,50 \mathrm{~b}$ & $40,00 \mathrm{~b}$ & $33,25 \mathrm{a}$ \\
\hline 6 & & $25,75 b$ & $26,50 \mathrm{~b}$ & $26,25 \mathrm{a}$ \\
\hline Testemunha & & $43,00 \mathrm{a}$ & $57,25 \mathrm{a}$ & $36,00 \mathrm{a}$ \\
\hline \multicolumn{5}{|c|}{$\begin{array}{l}\text { ** e * significativo a } 1 \text { e } 5 \% \text { de probabilidade pelo teste } \mathrm{F} \text {, } \\
\text { respectivamente; ns - não significativo a } 5 \% \text { de } \\
\text { probabilidade pelo teste F. Médias seguidas de mesma } \\
\text { letra não diferem entre si ao nível de } 5 \% \text { de probabilidade } \\
\text { pelo teste de Dunnett. }\end{array}$} \\
\hline
\end{tabular}



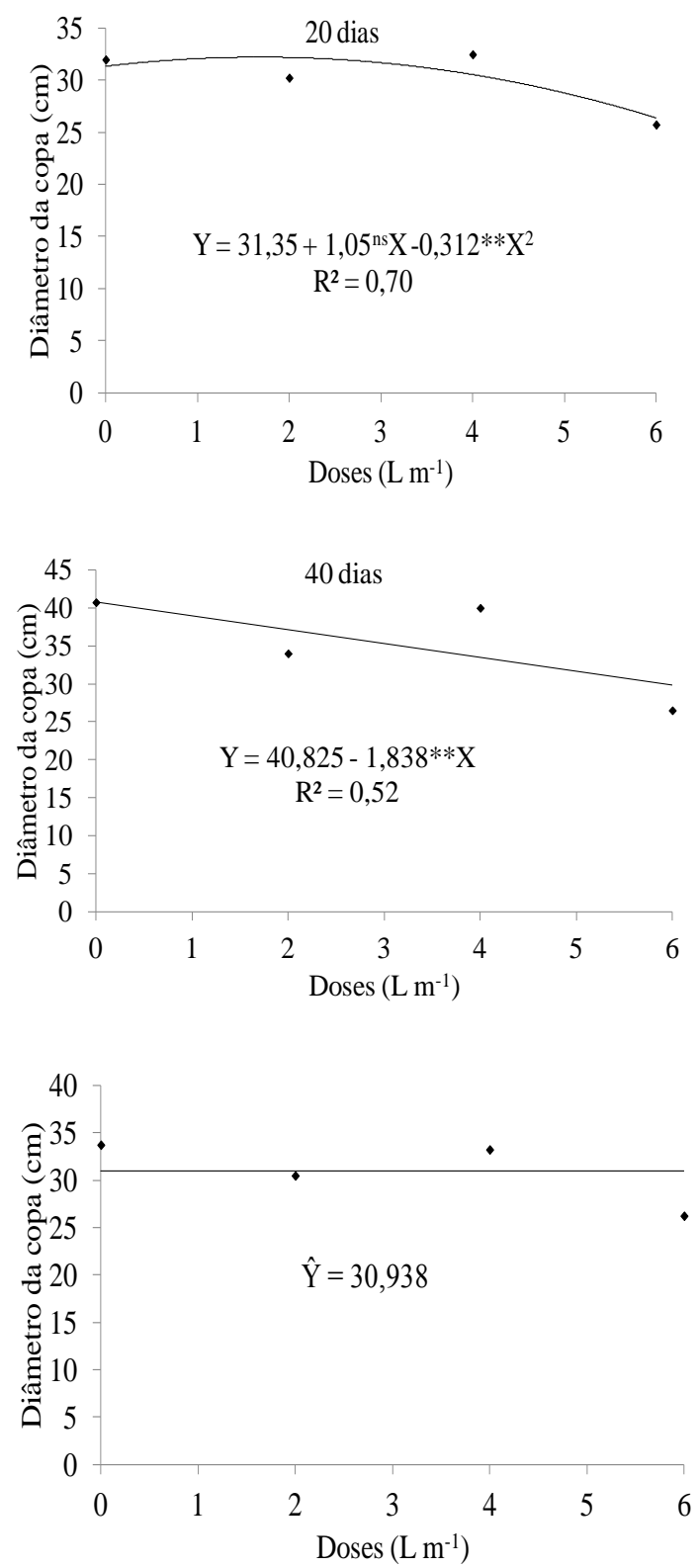

Figura 3. Diâmetro da copa do feijoeiro aos 20, 40 e 60 dias em função a diferentes doses do composto orgânico.

GALBIATTI et al. (2011), ao estudar o desenvolvimento do feijoeiro sob o uso de biofertilizante e adubação mineral, verificou-se que o uso dos dois compostos influenciaram no crescimento das plantas, na massa seca das folhas, caule, pecíolo, parte aérea e área foliar. Melém Junior et al. (2011) afirmam que os adubos orgânicos, além de fornecer nutrientes, o biofertilizante se destacam por exercer papel relevante, ou seja, o fornecimento de matéria orgânica para melhorar as propriedades físicas, químicas e biológicas do solo.

É possível que a inferioridade apresentada pela adubação com o composto orgânico, na maioria das variáveis, possa estar associada à relação Carbono/Nitrogênio, que era 42:1, ainda muito alta, o que pode ter interferido na absorção/nutrição da planta. A propósito, Villas Boas et al (2004), trabalhando com compostos orgânicos derivados de materiais com diferentes relação $\mathrm{C} / \mathrm{N}$, consideraram algumas hipóteses para explicar seus resultados. Assim, as relações $\mathrm{C} / \mathrm{N}$ entre os compostos são diferentes principalmente, considerando casca de eucalipto e serragem de madeira em relação à palhada de feijão.

Na palhada de feijão com menor relação C/N (10/1), a liberação de nutrientes é mais rápida em relação aos outros dois compostos. Esta inferência pode ser reforçada pelo fato que alguns compostos apresentam estruturas de difícil decomposição pela população microbiana do solo, como a celulose e lignina presentes nos compostos de casca de eucalipto e serragem de madeira. $\mathrm{O}$ material vegetal utilizado no presente trabalho era de alta relação $\mathrm{C} / \mathrm{N}$, pois mesmo depois do tratamento com ativadores biológicos ainda apresentavam relação $\mathrm{C} / \mathrm{N}$ igual a 42:1, muito alta ainda.

\section{CONCLUSÃO}

De acordo com os resultados obtidos, a adubação mineral foi mais responsiva em todas as variáveis analisadas em relação às diferentes doses de biofertilizante, nas condições em que o 
mesmo se encontrou quando aplicado em campo.

$\mathrm{O}$ adubo mostrou resultados bem diversificados entre as variáveis analisadas, e um efeito mais significativo nas doses de 2 e $4 \mathrm{~L}$, contudo não superou os resultados positivos da adubação mineral.

\section{REFERENCIAS BIBLIOGRÁFICAS}

OLIVEIRA, A.P; ARAUJO, J. S.; ALVES, E. U.; NORONHA, M. A. S.; CASSIMIRO, C. M.; MENDONÇA, F. G. Rendimento de feijãocaupi cultivado com esterco bovino mineral. Horticultura Brasileira, Brasília, v. 19, n. 1, p. 81-84, março, 2001.

GALBIATTI, J. A. et al. Desenvolvimento do feijoeiro sob o uso de biofertilizante e adubação mineral, São Paulo, Eng. Agríc. vol. 31 no.1 Jaboticabal jan./fev. 2011. Disponível em: 〈http://www.scielo.br/?lng=pt $>$. Acesso em: 22 jan. 2014.

BARROS, L.E.O.; LIBERALINO FILHO, J. Composto orgânico sólido e em suspensão na cultura do feijão Mungo verde (Vigna radiata. Wilkzeck). Mossoró: Revista verde de agroecologia e desenvolvimento sustentável, v.3, n.1, p.114-122, 2008. Disponível em: <http://www.gvaa.com.br/revista/index.php/RV ADS/article/viewFile/68/68 >. Acesso em: 07 jan. 2014

SILVA, R. P. et al. Efetividade de estirpes selecionadas para feijão caupi em solo da região semi-árida do sertão da Paraíba.
Recife: Revista Brasileira de Ciências Agrárias, v.3, n.2, p.105-110, 2008.

LIMA, J. G. A.; VIANA, T. V. A.; SOUSA, G. G.; WANDERLEY, J. A. C.; PINHEIRO NETO, L. G.; AZEVEDO, B. M. Crescimento inicial do milho fertirrigado com biofertilizante. Revista Agropecuária Científica no Semiárido, v.8, n.1, p.39- 44, 2012.

MELO, G.. M. P., MELO, V. P., MELO, W. J. Compostagem. Jaboticabal, Faculdade de Ciencias Agrarias e Veterinarias, 2007. 10p. Disponivel em: $<$ http://www.ambientenet.eng.br $>$. Acesso em: 17 jan. 2014.

SOUSA, G.G.; VIANA, T. V. A.; BRAGA, E; S.; AZEVEDO, B. M.; MARINHO, A. B.; BORGES, F. R. M. Fertirrigação com biofertilizante bovino: Efeitos no crescimento,trocas gasosas e na produtividade do pinhão manso, Revista Brasileira de Ciências Agrárias, v.8, n.3, p.503-509, 2013.

VILLAS BÔAS, R.L.; PASSOS, J.C.; FERNANDES, M.; BÜLL, L.T.; CEZAR, V.R.S.; GOTO, R. Efeito de doses e tipos de compostos orgânicos na produção de alface em dois solos sob ambiente protegido. Horticultura Brasileira, Brasília, v.22, n.1, p.2834, jan-mar 2004

MELÉM JÚNIOR, N. J.; BRITO, O. R.; FONSECA JÚNIOR, N. S.; FONSECA, I. C. B.; AGUIAR, S. X. Nutrição mineral e produção de feijão em áreas manejadas com e sem queima de resíduos orgânicos e diferentes tipos de adubação. Semina. Ciências Agrárias, v.32, n.1, p.7-18, 2011 